\title{
A Novel Fast Neutron Detector for Nuclear Data Measurements
}

I. Sagrado Garcia1, G. Ban ${ }^{1 *}$, V. Blideanu1, J. Blomgren², P. Eudes ${ }^{4}$, J.M. Fontbonne $^{1}$, Y. Foucher ${ }^{4}$, A. Guertin ${ }^{4}$, F. Hadad ${ }^{4}$, L. Hay ${ }^{1}$, A. Hildebrand ${ }^{2}$, G. Iltis ${ }^{1}$, C. Le Brun ${ }^{3}$, F.R. Lecolley1, J.F. Lecolley ${ }^{1}$, J.L. Lecouey ${ }^{1}$, T. Lefort ${ }^{1}$, N. Marie ${ }^{1}$, N. Olsson $^{2}$, S. Pomp ${ }^{2}$, M. Österlund ${ }^{2}$, A. Prokofiev ${ }^{5}$, J-C Steckmeyer ${ }^{1}$

1 Laboratoire de Physique Corpusculaire, IN2P3/CNRS, ENSICAEN, UCBN, 14050 Caen Cedex 04, France

2Department of neutron research, Uppsala University, Box 535, S-75120 Uppsala Sweden

3 Laboratoire de Physique Subatomique et de Cosmologie, IN2P3/CNRS, UJF, INPG,

38026 Grenoble Cedex, France

4 - SUBATECH, 44070 Nantes Cedex 03, France

5 The Svedberg Laboratory, Uppsala University,

Box 533, S-75121 Uppsala, Sweden

E-mail: banelpccaen.in2p3.fr

\begin{abstract}
Accelerator driven system will use a heavy element target such as lead. Many calculations are available to simulate high-energy spallation neutron induced reactions, but little data are available for comparison with the simulations.

In order to constrain the simulation tools we have measured $(\mathrm{n}, \mathrm{Xn})$ double differential cross section on different targets at The Svedberg Laboratory, Uppsala, Sweden. For neutron energy above $40 \mathrm{MeV}$, we have developed a novel detector, CLODIA, based on proton recoil and drift chambers to determine neutron energy. CLODIA (Chamber for LOcalization with DrIft and Amplification) is able to track recoil protons with energy up to $90 \mathrm{MeV}$ with spatial resolution of about one millimeter and a detection efficiency of $99 \%$ for each drift chamber. Using CLODIA coupled wit $^{*} \mathrm{~h}$ the SCANDAL set-up, we have been able to measure double differential $(\mathrm{n}, \mathrm{Xn})$ cross section on lead and iron for incident neutron energy in the $40-95 \mathrm{MeV}$ energy region.
\end{abstract}

International Workshop on Fast Neutron Detectors

University of Cape Town, South Africa

April 3-6, 2006

\section{${ }^{*}$ Speaker}




\section{Introduction}

Future accelerator-driven system (ADS) will couple an intense high-energy proton beam $(\sim 1 \mathrm{GeV}$, a few $\mathrm{mA})$ with a spallation target made of heavy element and a subcritical reactor core. The proton beam that is incident on the ADS target will create a large number of secondary particles, mainly neutrons, protons and other light charged particles, with energies covering the full range up to the $\mathrm{GeV}$ region. Although a large majority of the neutrons will be below $20 \mathrm{MeV}$, for safety reasons, as well as for code validation, the relatively small fraction at higher energies still has to be characterized.

Above $200 \mathrm{MeV}$ and under $20 \mathrm{MeV}$, the nuclear data are well documented [1], but in between (20-200 MeV), the lack of data makes ADS simulations less reliable. For inelastic $(\mathrm{n}, \mathrm{Xn})$ cross section in the $20-200 \mathrm{MeV}$ incident neutron energy region, very few measurements are available [2]. In order to obtain accurate data on $(\mathrm{n}, \mathrm{Xn})$ reactions, we have measured double differential cross section on different target and at different angles. We have been able to measure inelastic double differential $(\mathrm{n}, \mathrm{Xn})$ cross section $(\mathrm{E}, \Omega)$ on lead and iron for incident neutron energy of $96 \mathrm{MeV}$. This measurement was made possible by the development of a new detector named CLODIA, Chamber for LOcalization with DrIft and Amplification. This detector tracks the proton emitted after an $\mathrm{H}(\mathrm{n}, \mathrm{p})$ reaction in a polyethylene $\left(\mathrm{CH}_{2}\right)$ converter.

\section{Detector system}

\subsection{Detector requirements}

In order to define the incident energy of a neutron that causes the inelastic ( $n, X n)$ reaction, we detect a recoil proton produced in a hydrogenated converter. The proton energy and scattering angle allow us to calculate the initial neutron energy. The detector system has to be able to measure the proton trajectory and energy. Due to the naturally low flux of fast neutron beams $\left(\sim 4-5^{*} 10^{5} \mathrm{n} / \mathrm{cm}^{2} / \mathrm{s}\right)[3]$, the detector has to have high neutron to proton conversion efficiency. The detector has to have a good spatial (angular) resolution and high efficiency for recoil protons. It has to be able to reject charged particles besides of the recoil protons.

\subsection{CLODIA}

CLODIA is composed of eight $10 \times 10 \mathrm{~cm}^{2}$ detection modules stacked along the Z-axis, which coincides with the direction of a neutron scattered from the target. Seven converters are placed between modules in order to get neutron to proton conversion. Six of the converters are made of polyethylene $\left(\mathrm{CH}_{2}\right)$, and one is made of carbon, in order to subtract the carbon contribution in the inelastic part of the spectra. The thickness is $4 \mathrm{~mm}$ for the polyethylene converters and $2 \mathrm{~mm}$ for the carbon converter, in order to have equivalent energy losses. Each of the detection modules is an X-Y sensitive gas detector. The proton trajectory is reconstructed using $(\mathrm{X}, \mathrm{Y})$ position from each detector. Two different approaches are used to get the $(\mathrm{X}, \mathrm{Y})$ position. One is drift time, and the other one is resistive division.

The detection module is made of two Mylar foils, $2.5 \mu \mathrm{m}$ thick. The gap between the foils is $6.5 \mathrm{~mm}$. On each foil, 200 aluminum strips, each $150 \mathrm{~nm}$ thick and $880 \mu \mathrm{m}$ wide, are 
evaporated along the $\mathrm{Y}$ axis (Figure 1). The strips produce an electric drift field along the $\mathrm{X}$ axis. Voltage difference from one side to the other is about $4500 \mathrm{~V}$. We have chosen to use thin strips instead of wires, so that the protons "see" more homogeneous media along their travel. The charge is collected on a set of Ni wires, $50 \mu \mathrm{m}$, at the positive voltage side. The drift time is obtained by the (delayed) charge arrival, and a start is given by an external plastic scintillator located after the CLODIA detector (see Section 3).

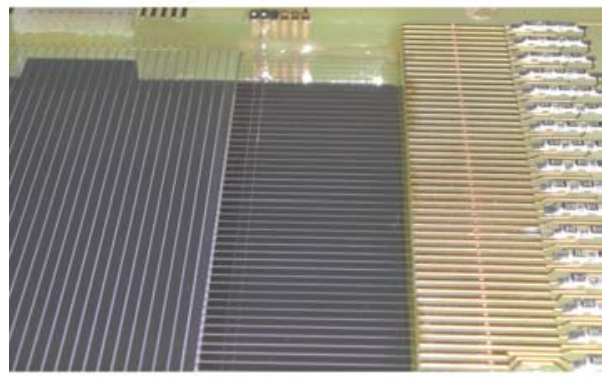

Figure 1. Detail of a CLODIA chamber on the left strips (vertical) for drift field and X localization, Strips (Horizontal) and CMS resistors for Y localization, the wire is seen near the middle of the picture

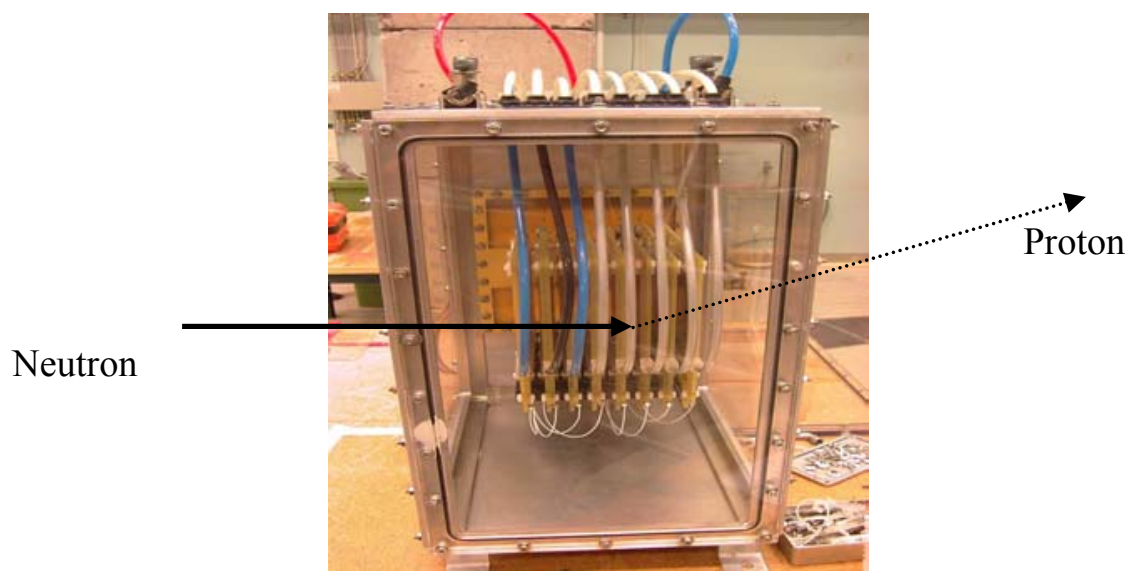

Figure 2. Overall view of CLODIA. Neutron comes from the left. Each module is viewed from the side and is set vertically. The tubes are gas distributor, and the white wires are HV distribution. The converters are not on the picture.

The potential along the electron path is between $-3000 \mathrm{~V}$ (the first strip) to $2000 \mathrm{~V}$ (anode). For Y localization, charge division is used. Around the anode, set at $2000 \mathrm{~V}$, an avalanche takes place. This point has been one of the most critical for CLODIA tuning. High voltage gives a large avalanche, but sparking is also favored by high voltage. A dedicated, very low noise preamplifier, located near the anode, have been developed in order to lower as much as possible the voltage used for the avalanche. From the start of development to the detector we actually use for measurement, the voltage has been lowered from $2000 \mathrm{~V}$ to $1500 \mathrm{~V}$. Near the anode, another set of 200 strips parallel to the $\mathrm{X}$ axis are evaporated, each of these strips are connected to the next one with a resistor. The charge division obtained with the resistive network gives the $\mathrm{Y}$ position. $\mathrm{P} 10$ gas $\left(\mathrm{Ar} 90 \%, \mathrm{CH}_{4} 10 \%\right)$ at $1 \mathrm{~atm}$ is flowing in each chamber. 
All chambers are placed in an aluminum box (Figure 2) with Mylar windows for particle entrance and exit and for detector maintenance.

\subsection{CLODIA performance}

For commissioning of CLODIA, we used a ${ }^{55} \mathrm{Fe} \mathrm{X}$-ray source. 5.4-keV X-rays produce the same amount of charge as $50-\mathrm{MeV}$ protons, so that we could experimentally simulate the signal produced by such protons. It is crucial to have good spatial resolution in order to track the recoil protons. We have measured the chambers' spatial resolution. The results give standard deviations $\sigma_{X}=0.3 \mathrm{~mm}$ and $\sigma_{Y}=0.7 \mathrm{~mm}$ along $X$ and $Y$ axes. Since we used at least three chambers, the proton diffusion angle is known with resolution that is sufficient for (n, Xn) measurement.

The CLODIA chamber is very sensitive to charged particles, and its efficiency for protons has been estimated as $99 \%$. Thus, the first detector can be used as a veto for charged particles coming from the target.

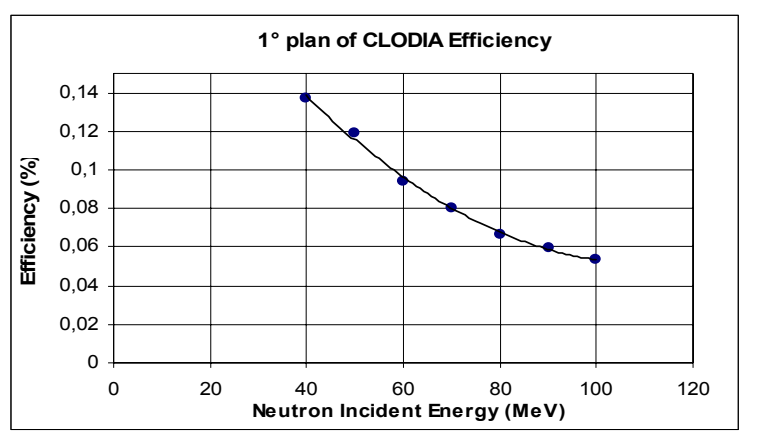

Figure 4. CLODIA efficiency versus incident neutron energy.

The detection principle of CLODIA is based on the elastic $\mathrm{H}(\mathrm{n}, \mathrm{p})$ reaction. This reaction has a small cross section value $(\sim 30 \mathrm{mb})$, and it was not possible to study it experimentally because of beam time limitations. Nevertheless, this reaction is well known, and simulation codes can be used to calculate the efficiency for the different planes of CLODIA. Figure 4 shows the efficiency of the first plane of CLODIA obtained using the GEANT3 code [4].

\section{Measurements}

\subsection{Set-up}

Inelastic $(\mathrm{n}, \mathrm{Xn})$ measurements were performed at The Svedberg Laboratory (TSL), where a $96 \mathrm{MeV}$ neutron beam is available. The schematic setup is shown on Figure 5. CLODIA is coupled with the SCANDAL device. The veto for charged particle rejection is obtained from a plastic scintillator, used jointly with the first CLODIA chamber.

The SCANDAL set-up [5] consists of a first trigger, two large wire chambers; and a second trigger followed by an array of CsI detectors. The coincidence between the two triggers gives the start for CLODIA acquisition. SCANDAL gives two additional points of the proton trajectory, and the recoil proton energy. The typical distance between the veto detector and the target is $1.40 \mathrm{~m}$. The solid angle is about $0.00262 \mathrm{sr}$. Due to all traversed materials, the proton energy measurement threshold in the CsI detectors is $40 \mathrm{MeV}$. 


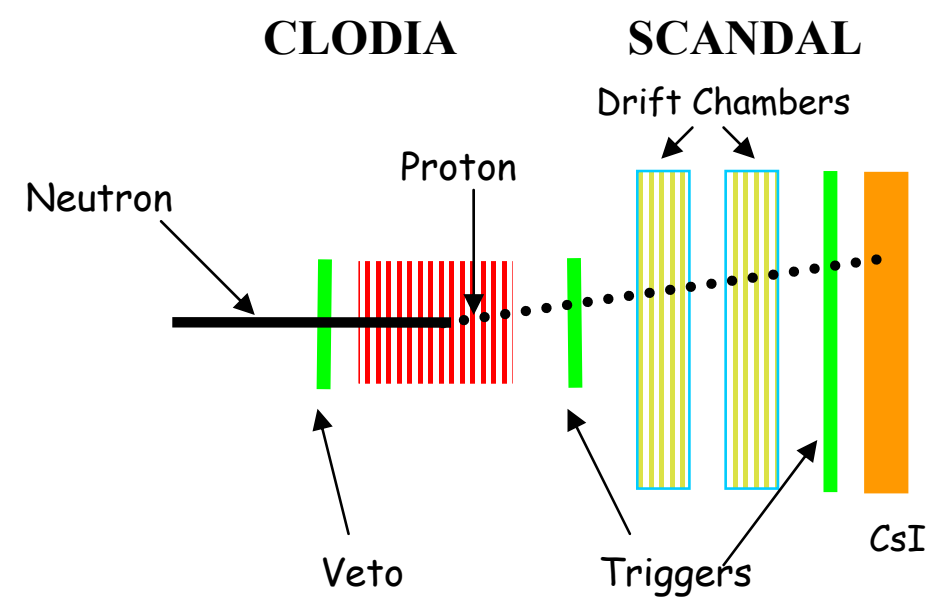

Figure 5. Experimental setup for $(\mathrm{n}, \mathrm{Xn})$ measurement at TSL

\subsection{Results}

Using CLODIA set up at TSL, two different experiments have been performed.

\section{$(\mathrm{n}, \mathrm{Xn})$ Measurement at $96 \mathrm{MeV}$}
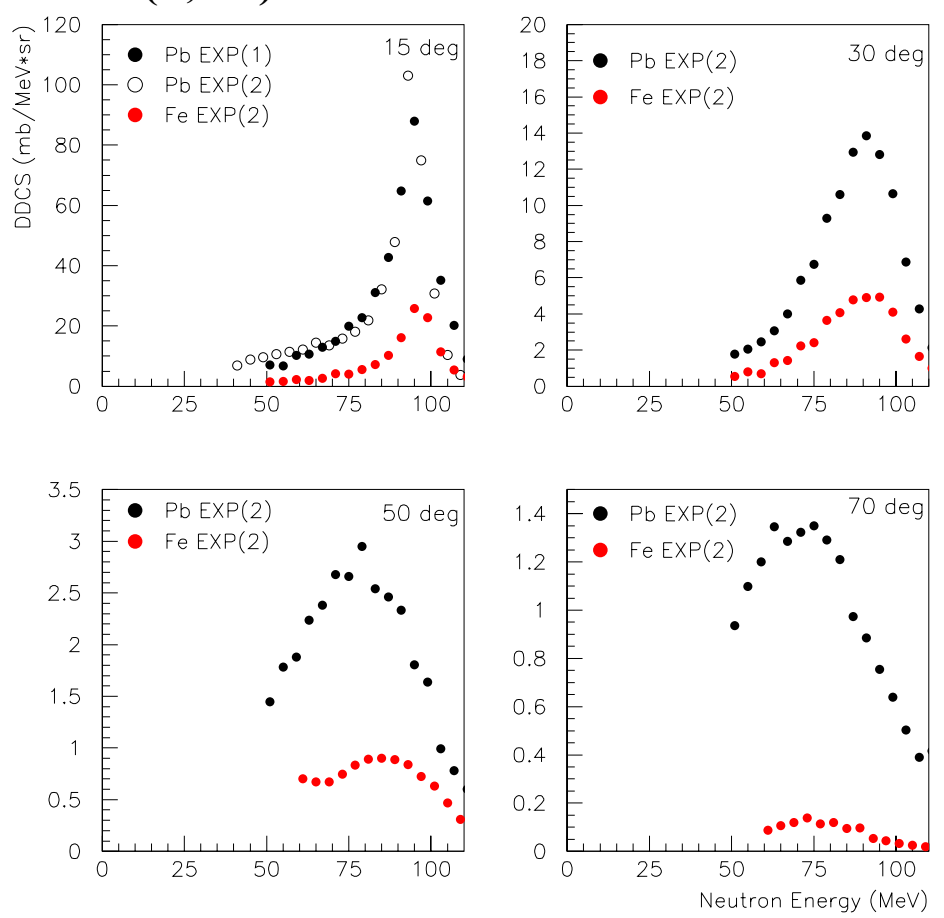

Figure 6 Double differential cross section obtained at different angles on lead and iron targets.

Due the low resolution in energy, the energy goes beyond the beam energy.

The first one was a feasibility test. During this test, we have measured the $(\mathrm{n}, \mathrm{Xn})$ cross section at $15^{\circ}$ for a lead target. After obtaining the first satisfactory results, we have performed 
the second experiment. We have been able to measure double differential cross section on lead and iron at $15^{\circ}, 30^{\circ}, 50^{\circ}$ and $70^{\circ}$ angles.

Figure 6 shows preliminary results. There is good agreement between the (n,Xn) cross sections at $15^{\circ}$ on lead during the two experiments. In addition, the elastic cross section $\mathrm{Pb}(\mathrm{n}, \mathrm{n})$ derived from our results is also in good agreement with other experimental measurements $[5,6]$ and with predictions of theoretical models [7]. The agreement between results of different experiments and calculations can be considered as verification of the whole experimental procedure.

\section{Conclusions}

The CLODIA detector coupled with the SCANDAL has allowed us to measure previously unknown $(\mathrm{n}, \mathrm{Xn})$ cross section on $\mathrm{Pb}$ and $\mathrm{Fe}$ for $40-96 \mathrm{MeV}$ incident neutrons. This series of measurement can be extended toward cross sections needed for heavy ion dosimetry where only a few data sets are available.

\section{References}

[1] HINDAS, High and Intermediate energy Nuclear Data for Accelerator-driven Systems, European Community, Contract No. FIKW-CT-2000-0031.

[2] E.L Hjort et al, Phys. Rev. C53 (1996) 237.

[3] S. Pomp et al., AIP Conf. Proc. 769, 780-783 (2005).

[4] GEANT3 User's guide.

[5] J. Klug et al, Nucl. Inst. Meth. in Phys. Res. A489 (2002) 282.

[6] G.L. Salmon et al, Nucl. Phys. 21 (1960) 15-20.

[7] A.J Koning, J.P. Delaroche, Nucl. Phys. A713, 231 (2003). 